\title{
COMPLEJO GRANITIICO SOLÍS DE MATAOJO: EVIDENCIAS DE MAGMATISMO SINCOLISIONAL RELACIONADO A SUBDUCCIÓN
}

\author{
PEDRO OYHANTCABAL *, MARÍA DERREGIBUS *, ROSSANA MUZIO**, SERGIO DE SOUZA* \\ e ELENA PEEL*
}

\begin{abstract}
SOLIS DE MATAOJO GRANITIC COMPLEX: EVIDENCES OF SINCOLLISIONAL MAGMATISM RELATED TO SUBDUCTION. The scope is to provide a model for the genesis and emplacement of the Solis de Matapjo Granitic Complex (SW Dom Feliciano Belt). A subsequent period to continental colusión, with generation of calk-alkaline magmatism related to oceanic crust dehydration, is proposed.

Keywords: Granitic magmatism, piuriserial magmatism, Brasiliano Cycle, Uruguay.
\end{abstract}

\begin{abstract}
RESUMEN En el extremo sur de la faja milonftica Sarandí del Yi - A* Solís Grande se ubica el Complejo Granítico Solís de Mataojo. Este Complejo, integrado por tonalitas, granodioritas y granitos está fuertemente deformado, con planaridad paralela a la foliación de las milonitas en las que se emplaza. Como modelo para la génesis y el emplazamiento del Complejo Granítico, se propone el emplazamiento sin-transcurrente durante el período subsiguiente a una colisión continental, con generación de magmatismo calco-alcalino vinculado a la
\end{abstract} deshidratación de corteza oceánica.

Palabras claves: Magmatismo granítico, magmatismo piuriserial, Ciclo Brasiliano, Uruguay.

ANTECEDENTES Desde Bossi et al (1967), se reconoce en el Uruguay la existencia de dos ciclos orogénicos diferentes. Los terrenos generados en la orogénesis más antigua, en lo que Almeida et al. (1973) denominan Ciclo Transamazónico (2,2 a 1,8 Ga), constituyen el Zócalo del Río de la Plata (Ferrando \& Fernández 1971) o Craton del Río de la Plata (Almeida et al 1973). Al este de una línea estructural, que va desde Isla Patrulla a Sierra de Animas (Ferrando \& Fernández 1971), se desarrollan los terrenos conformados durante el Ciclo Brasiliano (sensu Almeida 1971) y que Fragoso Cesar (1980) define como Cinturón Don Feliciano para el Escudo Uruguayo- Sur-Riograndense.

Preciozzi et al (1979) reconocen la importancia de la falla de Sarandí del Yí y plantean que este elemento permite subdividir el Zócalo del Río de la Plata en dos regiones.

Bossi \& Campal (1992) retoman este elemento estructural para definir tres grandes unidades en el Basamento del Uruguay.

Exceptuando la reciente contribución de Oyhant9abal et al (1992), los únicos antecedentes conocidos sobre el Complejo Granítico son Bossi (1966) quien hace referencia a la presencia de migmatitas en la zona y Umpierre \& Halpern (1971) quienes realizaron datación $\mathrm{Rb} / \mathrm{Sr}$ en material que definen como migmatitas del Puma (Lavalleja). La datación habría sido realizada sobre lo que en este trabajo se considera facies granodiorita a megacristales. La edad corregida es de $580 \mathrm{Ma}$ con razón inicial de ${ }^{87} \mathrm{Sr} /{ }^{86} \mathrm{Sr}$ de 0,704 . La granodiorita en ese afloramiento presenta signos de hidrotermalismo (venas de clorita, pirita, calcita) por lo que esa edad debería considerarse como mínima.

INTRODUCCIÓN En el área de ocurrencia del Complejo Granítico Solís de Mataojo (Fig. 1) confluyen la falla dextral Sarandí del Yi- A ${ }^{9}$ Solís Grande, representada en el área por una faja milonítica, y el "borde orogénico" brasiliano (Oyhantçabal et al 1993), lo que permite identifi- car dos zonas estructuralmente diferentes. La zona occidental del $\mathrm{A}^{\circ}$. Solís Grande está integrada por rocas correspondientes al Ciclo Transamazónico $(2 \pm 0,2 \mathrm{Ga})$, fundamentalmente milonitas e intrusiones graníticas sinpostectónicas. En la zona oriental, al este del $\mathrm{A}^{\mathrm{s}}$ Solís Grande se desarrollan estructuras con direcciones típicas del Cinturón Don Feliciano para el sur del Uruguay (NS-N30). Las litologías dominantes son milonitas en las que intruyó el Complejo Granítico Solís de Mataojo durante la reactivación sinestral de la falla mencionada, a fines del Ciclo Brasiliano.

PETROGRAFÍA Este Complejo presenta tres tipos litológicos principales: tonalitas, granodioritas y granitos; dispuestos en forma concordante con la geometría elongada de la intrusión (Fig. 2).

Tonalitas Presentan textura equigranular media y color gris oscuro. La mineralogía está caracterizada por plagioclasas sub-automorfas y zonadas (composición media An30), biotita ( $\mathrm{Ng}-\mathrm{Nm}$ marrón, $\mathrm{Np}$ amarillo-amarronado), anfíbol automorfo ( $\mathrm{Ng}$ verde-claro, $\mathrm{Nm}$ verde-pálido, $\mathrm{Np}$ amarillo), a veces clinopiroxenos corroídos con coronas de anfibol, cuarzo, esfenos xenomorfos a automorfos ocasionalmente incluidos en anfiboles, apatito relativamente abundante, alanita con bordes de epídoto, ortosa y circón. Los minerales secundarios son: clorita, calcita y epídoto. Se caracterizan por la fuerte orientación de las plagioclasas sub-automorfas, desarrollada durante la deformación en estadio magmático.

Granodioritas Presentan textura inequigranular porfiroide, con megacristales orientados de feldespato alcalino. La matriz es de grano medio y color gris-claro. Los megacristales son de microclina automorfa a sub-automorfa, de color blanco, con dimensiones medias de $5 \mathrm{~cm} \mathrm{x} 1,5 \mathrm{~cm}$,

\footnotetext{
* Facultad de Ciencias, Departamento de Geología, Tristán Narvaja 1674, CC 10773, Monte video 11200, Uruguay

** Facultad de Ciencias, Departamento de Geología, Tristán Narvaja 1674, CC 10773, Monte video 11200, Uruguay; Post-graduación UNESP, Caixa Postal 178, CEP 13500-970, Rio Claro, BRASIL
} 


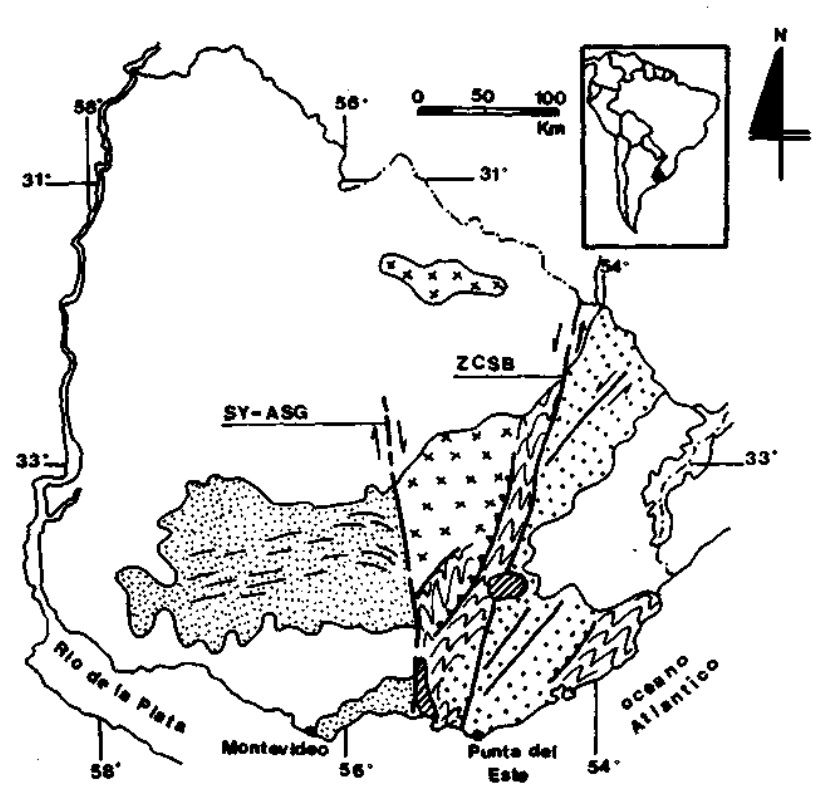

WiS Mognationo cimbrico

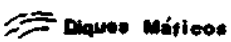

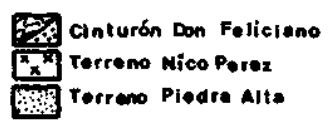

ZCSB _ Zoiada Cizalla da Siarra Ballena

SY-ASG_Falla Sarandi dal Vi-Arroyo Solis Grande

Figura 1 - Mapa de ubicación de los terrenos predevonianos del Uruguay (Preciozzi 1993, modificado)

Rgure 1 - Location map of Pre-Devonian terranes from Uruguay

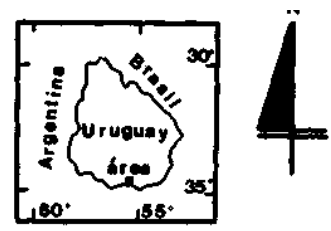

REFERENCIAS

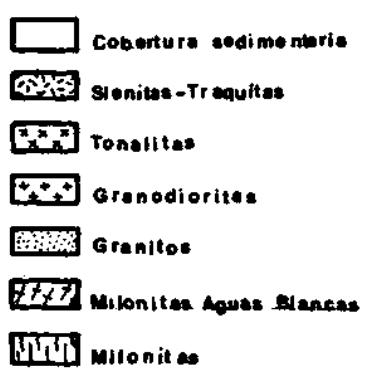

Siv Epesmente Proterozolce int.

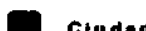

-or Hute necionales

(Preciozzi 1993, modified)

Figura 2 - Geología del área de estudio y distribución de las facies graníticas en el Complejo Granítico Solís de Mataojo

Figure 2 - Geology of the study área and distribution of granitic facies in the Solis de Mataojo Granitic Complex

constituyendo aproximadamente el $25 \%$ del volumen total de la roca. La mineralogía está compuesta por plagioclasas (An20) sub-automorfas, feldspato potásico (microclina) sub-automorfo, biotita (Ng-Nm marrón, $\mathrm{Np}$ amarillo- amarronado), anfíbol automorfo ( $\mathrm{Ng}$ verde-claro, $\mathrm{Nm}$ verde-pálido, Np amarillo), esfeno, apatito, alanita y circón. Como mineral secundario principal se destaca la clorita.

Granitos Se incluyen bajo esta denominación a un conjunto de rocas graníticas en sentido estricto, con biotita y/o muscovita como accesorios, con diferentes grados de deformación. La mineralogía está constituida por cuarzo, plagioclasa (An20), microclina, biotita, apatito, muscovita y circón. Los términos más deformados presentan abundantes neoformaciones de sericita. Texturalmente son isoxenomorfos, de grano fino a medio, con color gris-claro variando localmente para rosa-claro debido a alteración.

Enclaves Los enclaves son equigranulares finos a microgranulares, de composición cuarzo-diorítica y formas predominantemente elongadas de escala decimétrica. Están asociados a las facies tonalítica y granodiorítica, siendo su presencia restricta a ausente en los granitos. Los minerales principales son plagioclasas zonadas (oligoclasas), biotita (Ng-Nm marrón-rojizo, Np amarillo-amarronado), anfíbol ( $\mathrm{Ng}$ verde-claro, $\mathrm{Nm}$ verde-pálido, $\mathrm{Np}$ amarillo), cuarzo, apatito generalmente elongado a acicular, circón y esfeno (poco), en general formando cúmulos. Texturalmente se caracterizan por marcada orientación de las plagioclasas y minerales ferromagnesianos, y por la presencia de megacristales de plagioclasa, a veces conformando glomérulos.

ESTRUCTURAS DE DEFORMACIÓN El cuerpo presenta numerosas estructuras reconocibles tanto a escala microscópica como en afloramientos. A los efectos de ordenar la descripción, las mismas fueron separadas en aquellas resultantes de la deformación por procesos magmáticos y en estado sólido, siguiendo los criterios establecidos por Paterson et al. (1989).

Estructuras del estadio magmático Los minerales primarios, especialmente los cristales de plagioclasa subautomorfa y los megacristales de feldespato alcalino en las granodioritas, muestran entre sí marcada orientación subparalela. Pese a la existencia de deformación en estado sólido superpuesta, esta orientación se habría generado mayoritariamente durante la fase magmática.

- Los cristales de plagioclasa suelen aparecer imbricados, constituyendo indicador cinemático (Blumenfeld \& Bouchez 1988), demostrando en este caso el sentido sinestral de la deformación.

- Concentración de minerales ferromagnesianos en schlierens concordando con las estructuras descritas anteriormente.

- Los filones aplíticos tempranos aparecer plegados, no obstante parte de este plegamiento puede deberse a la deformación sub-solidus.

Estructuras de estado sólido Los minerales muestran numerosas evidencias de deformación plástica como: extinción ondulante en cuarzo, maclas torcidas en plagioclasas, kinks en biotitas, formación de subgranos y recristalización de cuarzo. Los procesos de recuperación que conducen a la recristalización del cuarzo dan como resultado la frecuente ocurrencia de texturas mortero.

- Es observable, junto con la reducción del tamaño de grano del cuarzo, la elongación de los granos recristalizados resultando microestructuras tipo ribbons, agregados lenticulares de muscovita y abundantes lentes de mirmequitas.

- La deformación que determina la planaridad que presenta el cuerpo comenzó en el estadio magmático y hacia el final de la cristalización la misma se desarrolla en forma heterogénea, concentrándose en fajas miloníticas de espesor centimétrico a hectamétrico. Estas fajas miloníticas des- 
plazan a filones aplíticos tardios plegados con una componente sines trai.

- La presencia de feldespatos con bordes fuertemente corroídos dentro de Ias fajas más milonitizadas puede deberse ai calor liberado por thermal feed-back, generando cierta fusion y acelerando Ia absorción de Ia deformación a través de estas fajas. Es frecuente también Ia fractura y boudinage de estos minerales, con recristalizaciones de cuarzo, mica, clorita y calcita en Ias zonas deformadas. Esto es debido a que a bajas temperaturas (p.ex. facies esquistos verdes), los feldespatos solo tienden a fracturarse (Simpson 1985).

- Las sombras de destrucción y de presión en los megacristales de feldespatos indican sentido de movimiento sinestral.

- Ocasionalmente, se observan diaclasas tensionales (en echellon) rellenas de cuarzo que confirman ei desplazamiento senalado anteriormente.

Modelo de emplazamiento Un conjunto de evidencias senalan emplazamiento sintectónico en una zona de cizalla transcurrente:

- geometria elongada con eje mayor paralelo a Ia faja cizallada;

- los diferentes tipos litológicos se disponen paralelos a Ia elongación dei cuerpo;

- Ia foliación magmática y Ia foliación tectónica dei complejo granítico son paralelas a Ia foliación tectónica de Ias encajantes;

- ei cuerpo es composicionalmente heterogêneo con abundantes xenolitos; $y$

- Ias lineaciones de estiramiento mineral son sub-horizontales.

Estos elementos han sido senalados por Hutton (1988), Paterson et ai. (1990) y Morand (1992) como indicadores de emplazamiento asociado a cizallamiento transcurrente.

GEOQUíMICA DEL COMPLEJO El estúdio de Ia geoquímica dei Complejo busca elucidar Ia hipótesis planteada por Oyhantçabal et ai. (1992): magmatismo en margen continental active vinculado a una tectónica transcurrente.

Se analizaron los elementos mayores en muestras de Ias três facies identificadas en ei Complejo y en los filones ácidos. La identificación de Ias diferentes muestras y los resultados están presentados en ei cuadro 1.

En primer lugar, fue confirmada Ia clasificación petrológica de Ias muestras a través de un diagrama RI - R2 (De la Roche et ai. 1980), el cual no se publica por no presentar información relevante. La localización de dichas muestras en un diagrama R1-R2 (Batchelor \& Bowden 1985), que relaciona composición química con ambiente tectónico se encuentra en Ia figura 3.

La distribución de los puntos representativos de Ias diferentes muestras confirma Ia tendência ya encontrada de magmatismo en margen continental activo sobre el fin del proceso de subducción. Se estúdio Ia distribución de Ias muestras en los diagramas de Maniar \& Piccoli (1989). Si bien, en esos diagramas, aparecen agrupadas entre los granitoides de arco islã, arco continental o colisión continental, no es posible distinguir entre esos tipos. Según esos autores serían Ias relaciones molares de $\mathrm{Al}_{2} \mathrm{O}_{3}$ / $\left(\mathrm{Na}_{2} \mathrm{O}+\mathrm{K}_{2} \mathrm{O}+\mathrm{CaO}\right)$ que permitirían hacerlo. En ei cuadro 1 , se presentan los valores de esas relaciones para todas Ias muestras que aunque oscilan entre 0,61 y 1,21 , no permiten obtener nitidamente los grupos de valores claves (menor que 1,05 para granitos de arco islã y arco continental o mayor que 1,15 para granitos de colisión continental).

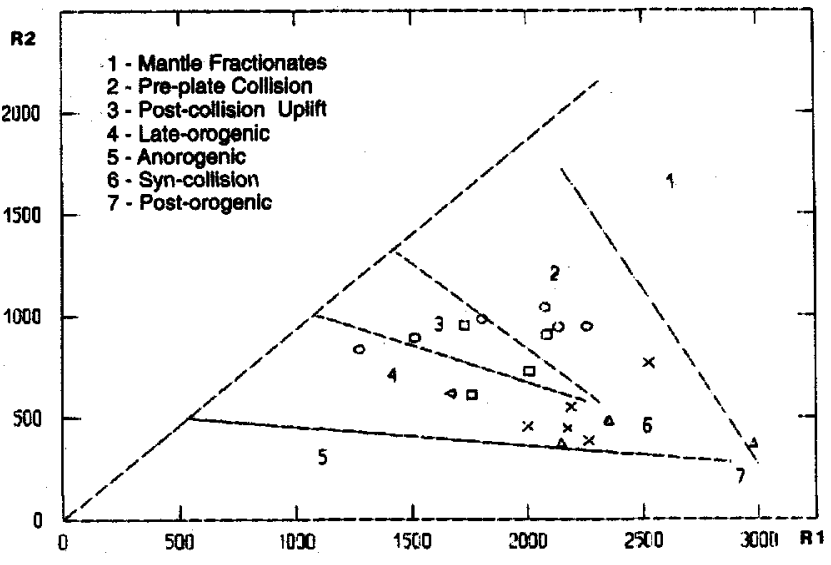

Figura 3 - Diagrama RI - R2 (Batchelor \& Bowden 1985). Símbolos: $O=$ tonalitas $; \square=$ granodioritas; $x=$ granitos; $\triangleleft=$ filón en tonalitas; $\Delta=$ filones en granodioritas

Figura 3 - R1 - R2 diagram (Batchelor \& Bowden 1985). Symbols: o = tonalites; $\square=$ granodiorites; $\mathbf{x}=$ granites; $\Delta=$ dikes in tonalites; $\triangle=$ dikes in granodiorites

En la figura 4 se representa ei índice de Shand que corresponde a Ia representación gráfica de Ias relaciones molares anteriormente mencionadas. La mayoría de Ias muestras quedan localizadas en ei campo de los granitos metaluminosos. Se observa también que algunos de los términos más diferenciados están localizados en ei campo de los peraluminosos; es Ia zona definida por Maniar \& Piccoli (1989) como de los granitos de arco continental o de

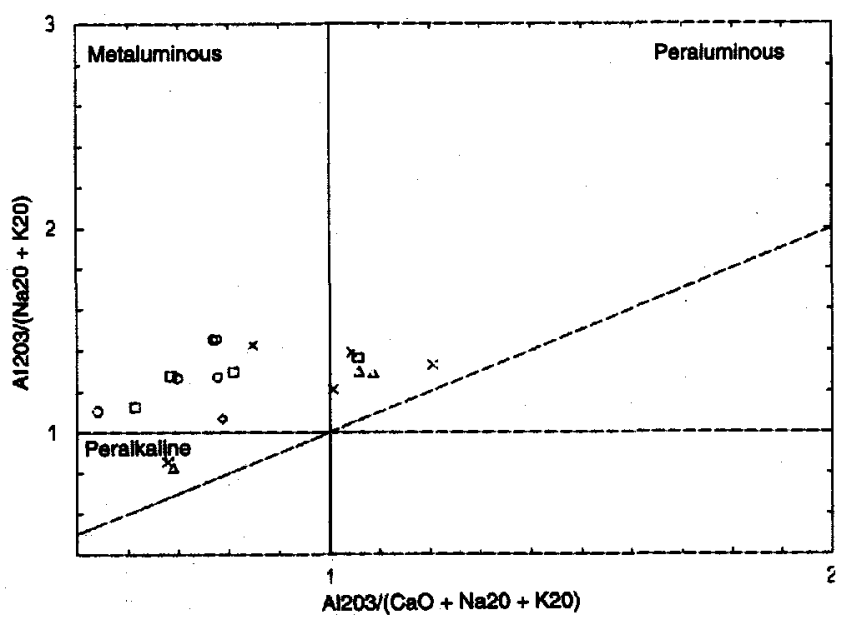

Figura 4-Indice de Shand con relaciones molares. Símbolos como en la figura 3

Figure 4 - Shand's indices with molar relations (Maniar \& Piccoli 1989). Same symbols as figure 3

colisión continental.

Se estúdio también, ei comportamiento de las muestras en ei diagrama propuesto por Brown (1982), que relaciona \% $\mathrm{SiO}_{2}$ contra log $\% \mathrm{CaO} /\left(\% \mathrm{Na}_{2} \mathrm{O}+\% \mathrm{~K}_{2} \mathrm{O}\right)$ (Fig. 5). Este autor hace referencia a Ia relación entre composición química y madurez dei arco magmático; Ia zona correspondiente a los procesos de subducción más avanzados (granitoides de Nueva Guinea, magmatismo shoshonítico de alto K) está representada por lã línea inferior en tanto que Ia superior corresponde a Ia de los granitos calco-alcalinos. La nube de puntos representativos de Solís de Mataojo se localiza en Ia zona de arcos maduros entire los granitos calco-alcalinos, no alcanzando ei limite de los correspondientes ai magmatismo shoshonítico. 
Cuadro 1-Análisis químicos de elementos mayores.(Muestras 17-1 a 135-1: tonalitas; 18-1 a 22-1: granodioritas; 31-2 a 591: granitos; 164-1: filón en tonalita; 27-1 a 34-1: filones en granodioritas)

Chart 1 - Major elements chemical analysis. (Samples 17-1 to 135-1: tonalites; 18-1 to 22-1: granodiorites; 31-2 to 59-1: granites; 164-1: dike in tonalite; 27-1 to 34-1: dikes in granodiorites)

\begin{tabular}{|c|c|c|c|c|c|c|c|}
\hline NoMUE & $17-1$ & 20-1 & $28-1$ & $47-2$ & $53-1$ & $135-1$ & \\
\hline$\% \mathrm{SiO} 2$ & 65,11 & 63,78 & 63,47 & 62,17 & 64,33 & 65,74 & \\
\hline$\% \mathrm{TiO}_{2}$ & 0,49 & 0,80 & 0,46 & 0,47 & 0,49 & 0,45 & \\
\hline \%Al2O3 & 13,73 & 11,29 & 13,40 & 14,61 & 10,51 & 13,31 & \\
\hline$\% \mathrm{FeO}(\mathrm{t})$ & 5,61 & 7,56 & 4,56 & 5,01 & 5,21 & 4,88 & \\
\hline$\% \mathrm{MnO}$ & 0,11 & 0,14 & 0,10 & & 0,13 & 0,11 & \\
\hline $9 \mathrm{MgO}$ & 3,70 & 2,81 & 4,24 & 3,50 & 4,95 & 4,09 & \\
\hline$\% \mathrm{CaO}$ & 4,56 & 4,40 & 4,75 & 4,01 & 5,48 & 4,49 & \\
\hline$\% \mathrm{Na} 2 \mathrm{O}$ & 4,01 & 5,40 & 4,59 & 4,13 & 4,20 & 4,12 & \\
\hline$\% \mathrm{~K} 20$ & 2,61 & 3,52 & 2,80 & 4,33 & 2,40 & 2,19 & \\
\hline$\% \mathrm{P} 205$ & 0,03 & & 0,14 & & 0,06 & & \\
\hline$\% \mathrm{H} 2 \mathrm{O}(-)$ & 0,21 & 0,21 & 0,35 & 0,25 & 0,25 & 0,35 & \\
\hline$\% \mathrm{H} 2 \mathrm{O}(+)$ & 0,56 & 0,04 & 0,06 & & 0,20 & & \\
\hline TOTAL & 100,7 & 100,0 & 98,9 & 98,5 & 98,2 & 99.7 & \\
\hline $\mathbf{R}$ & 0,78 & 0,55 & 0,70 & 0,78 & 0,54 & 0,77 & \\
\hline NoMUE & $18-1$ & $18-2$ & $18-3$ & $22-1$ & $31-2$ & $32-1$ & $52-2$ \\
\hline$\% \mathrm{SiO}_{2}$ & 63,88 & 64,77 & 65,56 & 69,23 & 70,54 & 69,91 & 73,79 \\
\hline \%TIO2 & 0,81 & 0,38 & 0,56 & 0,34 & 0.43 & 0,19 & 0,33 \\
\hline \%Al2O3 & 12,19 & 12,94 & 12,57 & 17,49 & 13,51 & 15,43 & 11,35 \\
\hline$\% \mathrm{FeO}(\mathrm{t})$ & 6,00 & 5,15 & 5,14 & 2,54 & 4,36 & 2,42 & 1,52 \\
\hline$\% \mathrm{MnO}$ & 0,13 & 0,13 & 0,11 & & 0,11 & 0,05 & 0,04 \\
\hline$\% \mathrm{MgO}$ & 3,68 & 2,36 & 3,15 & 0,98 & 2,38 & 0,62 & 0,58 \\
\hline$\% \mathrm{CaO}$ & 4,96 & 3,31 & 4,68 & 2,04 & 3,57 & 2,02 & 1,92 \\
\hline $9 \mathrm{Na} 2 \mathrm{O}$ & 4,20 & 3,91 & 3,80 & 5,33 & 3,92 & 4,50 & 4,52 \\
\hline$\% \mathrm{~K} 20$ & 3,62 & 3,27 & 3,32 & 3,76 & 3,49 & 3,41 & 5,38 \\
\hline$\%$ P205 & 0,08 & 0,08 & & & 0,16 & 0,04 & 0,12 \\
\hline$\% \mathrm{H} 2 \mathrm{O}(-)$ & 0,22 & 0,35 & 0,20 & 0,21 & 0,02 & 0,35 & 0,17 \\
\hline$\% \mathrm{H} 2 \mathrm{O}(+)$ & 0,28 & 0,28 & & & & 0,01 & \\
\hline TOTAL & 100,1 & 96,9 & 99,1 & 101,9 & 102,5 & 99,0 & 99,7 \\
\hline $\mathbf{R}$ & 0,61 & 0,81 & 0,68 & 1,06 & 0,81 & 1,04 & 0,68 \\
\hline NoMUE & $59-1$ & $100-1$ & $164-1$ & $27-1$ & $31-1$ & $34-1$ & \\
\hline$\% \mathrm{SiO} 2$ & 72,87 & 73,00 & 70,35 & 74,76 & 74,24 & 77,61 & \\
\hline \%TIO2 & 0,15 & 0,07 & 0,33 & 0,35 & 0,11 & 0,22 & \\
\hline \%Al203 & 15,59 & 14,59 & 14,38 & 15,57 & 10,65 & 12,63 & \\
\hline$\% \mathrm{FeO}(\mathrm{t})$ & 2,00 & 2,91 & 3,56 & 0,94 & 0,55 & 2,51 & \\
\hline$\% \mathrm{MnO}$ & 0,04 & & & 0,04 & 0,06 & 0,03 & \\
\hline$\% \mathrm{MgO}$ & 0,18 & 0.36 & 1,03 & 0,39 & 0.29 & 0,31 & \\
\hline$\% \mathrm{CaO}$ & 0,67 & 1,34 & 2,64 & 1,48 & 1,42 & 1,01 & \\
\hline$\% \mathrm{Na} 2 \mathrm{O}$ & 4,07 & 4,30 & 5,39 & 4,83 & 4,41 & 2,77 & \\
\hline$\% \mathrm{~K} 20$ & 4,64 & 4,61 & 4,24 & 3,75 & 5,20 & 4,83 & \\
\hline \%P205 & 0,05 & & & 0,04 & & 0,04 & \\
\hline$\% \mathrm{H} 2 \mathrm{O}(-)$ & 0.34 & 0,25 & 0,12 & 0,23 & 0,27 & 0,18 & \\
\hline$\% \mathrm{H} 2 \mathrm{O}(+)$ & & & & 0,00 & 0,08 & 0,27 & \\
\hline TOTAL & 100,6 & 101,4 & 102,0 & 102,4 & 97,3 & 102,4 & \\
\hline $\mathbf{R}$ & 1,21 & 1,01 & 0.79 & 1,06 & 0,69 & 1,09 & \\
\hline \multicolumn{8}{|c|}{$\mathrm{R}=\mathrm{AI} 203 /(\mathrm{K} 20+\mathrm{Na} 20+\mathrm{Ca} 0)$} \\
\hline
\end{tabular}




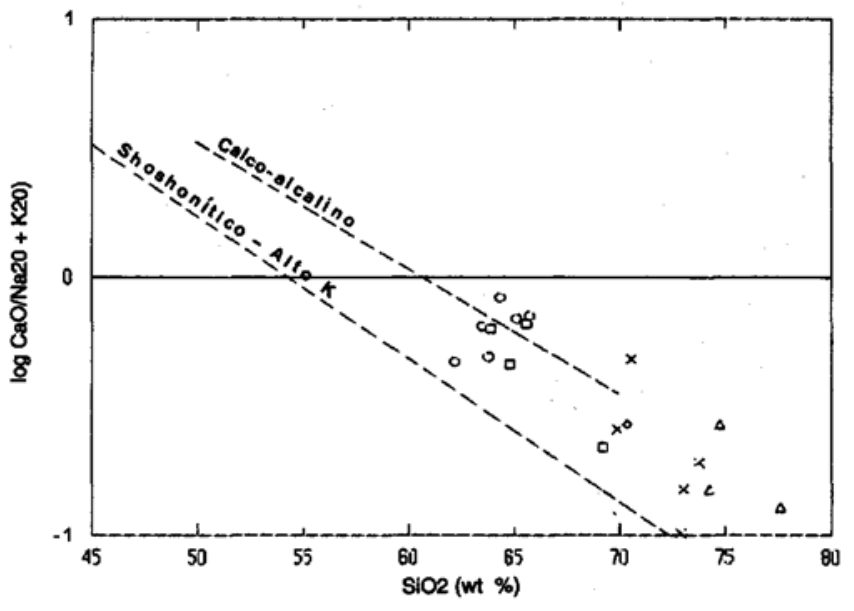

Figura 5 - Diagrama de Brown (J982). Misma simbología de Ia figura 3

Figure $5-\mathrm{SiO}_{2}$ versus $\log \mathrm{CaO} /\left(\mathrm{Na}_{2} \mathrm{O}+\mathrm{K}_{2} \mathrm{O}\right)$ diagram (Brown 1982). Same symbols as figure 3

Si se representar! las muestras en un diagrama de Rogers \& Greenberg (1990) (Fig. 6), se les encuentra en su mayoria en la zona de los granites calco-alcalinos.

Aunque no ha sido aún mencionado, Ia geoquímica corrobora la clasificación de este Complejo como de Tipo I y Ia representación en un diagrama de Marker permite caracterizarlo como perteneciente a un magmatismo alto K (Wilson 1989).

CONCLUSIONES De acuerdo con las evidencias expuestas, Ia implantación dei Complejo está vinculado a una zona de cizallamiento transcurrente con sentido sinestral. Fragoso César et ai. (1990) plantean que Ias zonas de cizallamiento sub-verticales con intrusiones asociadas se desarrollan durante Ia etapa colisional tardia; Fernandes et ai (1992) indican zonas de transcurrencia sinestral asociadas a magmatismo alto $\mathrm{K}$ durante Ia fase cinemática K2. Lo anteriormente expresado permite inferir que ei emplazamiento dei Complejo debe haber ocurrido durante Ia etapa colisional tardia.

La geoquímica de los elementos mayores indica que se está en presencia de magmatismo calco-alcalino de alto

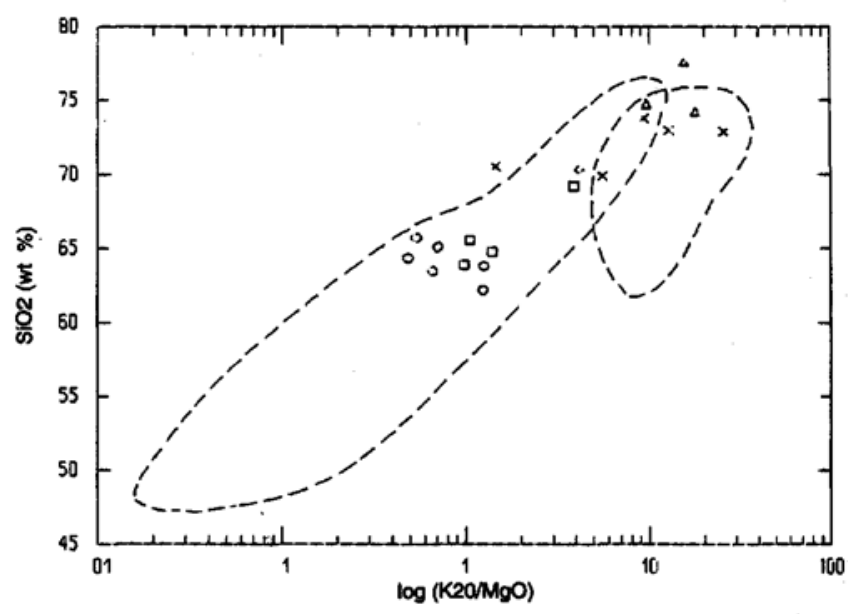

Figura 6 - Diagrama de Rogers \& Greenberg (1990). Misma simbología de Ia figura 3

Figure 6 - Rogers \& Greenberg's diagram (1990). Same symbols as figure 3

$\mathrm{K}$ que estaria relacionado a subducción en ambiente de arco maduro para Ias facies tonalítica y granodiorítica, y de magmatismo alcali-cálcico en ei caso de los granitos.

Lãs evidencias estructurales indican ei emplazamiento dei cuerpo en Ia etapa colisional tardia en tanto que Ias evidencias geoquímicas y petrográficas presentadas por Ias tonalitas y granodioritas indican magmatismo vinculado a subducción. Los granitos por su parte tienen características diferentes, sugiriendo Ia posibilidad de corresponder a una intrusion independiente más joven, Io que caracteriza ai complejo como pluriserial.

Confrontando Io senalado anteriormente con Io postulado por Bonin (1988), se puede establecer como modelo para Ia gênesis y emplazamiento dei Complejo Granítico Solís de Mataojo ei correspondiente ai período subsiguiente a Ia colisión continental. Tal como ei mismo autor To establece, en esa etapa se siguen generando magmas calco-alcalinos vinculados a Ia deshidratación de Ia corteza oceânica. Este período puede extenderse hasta $50 \mathrm{Ma}$ una vez finalizados los procesos de subducción.

\section{REFERENCIAS BIBLIOGRÁFICAS}

ALMEIDA, F. 1971. Geochronological division of the Precambrian of South America. Rev. Bras. Geoc., 1:3-21.

ALMEIDA, F.; AMARAL, G.; CORDANI, U.; KAWASHITA, K. 1973. The Precambrian evolution of the South American cratonic margin south of the Amazon river. In: NAIRM, A.; STEHLI, F. ed. The Ocean Basin and Margins. New York, Plenum Press, p. 441-446

BATCHELOR, R. \& BOWDEN, P. 1985. Petrogenetic interpretation of granitoid rock series using multicationic parameters. Chem. Geol., 48:43-55.

BLUMENFELD, P. \& BOUCHEZ, J.L. 1988. Shear criteria in granite and migmatite deformed in the magmatic and solid states. /. Struct. Geol., 10(4):361-372.

BONIN, B. 1988. From erogenic to anorogenic environments: evidence from associated magmatic episodes. Schweit. Mineral. Petrogr. Mitteil. 68:301-311.

BOSSI, J. 1966. Geologia del Uruguay. Departamento de Publicaciones UDELAR, $455 \mathrm{p}$.

BOSSI, J.; FERRANDO, L.; ALBANELL, H. 1967. Basamento cristalino del sureste del Uruguay. EOS, 53:2-10

BOSSI, J. \& CAMPAL, N. 1992. Magmatismo y tectónica trascurrente durante el Paleozoico Inferior en Uruguay. In: GUTIERREZ, J.; SAAVEDRA, J.; RABANO, I. ed. Paleozoico Inferior en Ibero-América. Extremadura, Universidad de Extremadura. p. $343-356$

BROWN, G. 1982. Calc-alkaline intrusive rocks: their diversity, evolution and relation to volcanic arcs. In: THORPE, R. ed. Andesites. Wiley \& Sons. p. 437-461.
DE LA ROCHE, H.; LETERRIER, J.; GRANCLAUDE, P.; MARCHAL, M. 1980. A classification of volcanic and plutonic rocks using R1-R2 diagram and major element analyses its relationships with current nomenclature. Chem. Geol., 29:183-210.

FERNANDES, L.; TOMMASI, A.; PORCHER, C. 1992. Deformation patterns in the southern Brazilian branch of the Dom Feliciano Belt: A reappraisal. 3. South Am. Earth Sci., 5(1):75-96.

FERRANDO, L. \& FERNANDEZ, A. 1971. Esquema tectónico-cronoestratigráfico del Predevoniano en el Uruguay. In: CONOR. BRAS. GEOL., 25. São Paulo, 1971. Anais... São Paulo, SBG. v. 1, p. $199-210$.

FRAGOSO CESAR, A.R.S. 1980. O Cráton Rio de la Plata e o Cinturão Dom Feliciano no Escudo Uruguaio - Sul Riograndense. In: CONGR. BRÁS. GEOL., 31. Camboriú, 1980. Anais... Camboriú, SBG. v. 5, p. 2879-2892.

FRAGOSO CESAR, A.R.S.; MACHADO, R.; MONTEIRO, R.; SALLET, R. 1990. Nappes e estruturas correlatas do Cinturão Dom Feliciano no Escudo Uruguaio-Sul-Riograndense: uma introdução ao proble-

ma. Acta Geol Leopold., 13(30):75-92.

HUTTON, D.H.W. 1988. Granite emplacement mechanisms and tectonic controls:inferences from deformation studies. Trans. R. Soc. Edinburgh, 79:245-255.

MANIAR, P. \& PICCOLI, P. 1989. Tectonic discrimination of granitoids. Geol. Soc. Am. Bull., 101:635-643.

MORAND, V.J. 1992. Pluton emplacement in strike-slip fault zones: the Doctors Flat Pluton, Victoria, Australia. J. Struct. Geol., 14(2): 205-213. 
OYHANTCABAL, R: DERREGIBUS, M · SOUZA, S ; MUZIO, R 1992. O Complexo Granftico Solís de Mataojo (Uruguai) no extremo SW do Cinturão Dom Feliciano. In: CONOR. BRAS. GEOL., 37. São Paulo, 1992. Boletim de Resumos Expandidos... São Paulo, SBG. v. 1, p. 400-401.

OYHANTÇABAL, R; SOUZA, S.; MUZIO, R. 1993. Geologia y aspectos estructurales dei borde orogénico en ei extremo sur dei Anturón Don Feliciano. In: SIMP. INTERN. DEL NEOPROTEROZÓICOCAMBRICO DE LA CUENCA DEL PLATA, I. Las Palomas-Minas, 1993. Boletim de Resumos Expandidos... Lãs Palomas-Minas, Uruguay, DI.NA.MI.GE. Tomo 1, resumo $\mathrm{n}^{\mathrm{a}} 27$.

PATERSON, S.; VERNON, R.; TOBISCH, O. 1989. A review of criteria for the identification of magmatic and tectonic foliations in granitoids. J. Struct. Geol, 11(3):349-363.

PATERSON, S.; TOBISCH, O.; MORAND, V. 1990. The influence of large ductile shear zones on the emplacement and deformation of the Wyangala Batholith, SE Australia. /. Struct. Geol., 14(2):205-213.

PRECIOZZI, F. 1993. Petrography and Geochemistry of Five Granitic Plutons from South-Central Uruguay: Contribution to the Knowl- edge of the Piedra Alta Terrane. Quebec. 200 p. (Ph.D.Thesis, Université du Quebec à Montreal).

PRECIOZZI, F.; SPOTURNO, J.; HEINZEN, W. 1979. Carta GeoEstructural del Uruguay. Montevideo, DI.NA.MI.GE. 62 p.

ROGERS, J. \& GREENBERG, J. 1990. Late erogenic, post erogenic and anorogenic granites: distinction by major element and trace element chemistry and possible origins. J. Geol., 98(3):291-309.

SIMPSON, C. 1985. Deformation of granitic rocks across the brittleductile transition. J. Struct. Geol, 7(5):503-511.

UMPIERRE, M. \& HALPERN, M. 1971. Edades estroncio-rubidio en rocas cristalinas del sur de Ia República Oriental del Uruguay. Asoc. Geol. Argent. Rev., 26(2):133-151.

WILSON, M. 1989. Igneous Petrogenesis. London, Unwin Hyman Inc. $456 \mathrm{p}$.

MANUSCRITO A795

Recebido em 13 de setembro de 1993

Revisão do autor em 28 de julho de 1994 Revisão aceita em 31 de agosto 